\title{
Identification of Brain Tumor using Image Processing and Neural Networks
}

\author{
Vanshika Dhillo ${ }^{1}$ \\ ${ }^{1}$ Student, Dept. of E\&TC, \\ MIT Academy of Engineering, Pune, India
}

\begin{abstract}
Machine learning, evolution computation, vision, robotics, speech processing, natural language process and planning are some of the building blocks of artificial intelligence (AI). Over the years AI has developed a lot and contributed its own share to make human life easy and compact. Technology happens to be a term with various definitions and it keeps changing with time. From a computer as big as a room to a computer on your finger tips technology has developed and so has the human race. The need of the time is quick results with accurate answers in the easiest way possible. AI is the current most developing field and has shown some extra ordinary results which have proved its importance. Use of AI has been adapted in almost all fields and where ever possible. Neural network is one of the small components under this big umbrella of artificial intelligence. Medical field has also developed itself over the time and has increased the life expectancy. The entire idea behind this project is to use neural networks and the medical knowledge hand in hand.
\end{abstract}

Keywords - Image processing, neural networks, biomedical, artificial intelligence, brain tumor

\section{INTRODUCTION}

Over the past few decades medical studies have adapted a lot of changes in their approach towards treating a patient which has improved the standards of living and provided comfort. Biomedical tools have been developed which help the doctors to analyze and study about a particular disease or disorder. Even after so many efforts and thrive there are some life taking afflictions like cancer, leukemia or brain tumor which if not detected on time can be proved as fatal.

According to a survey taken by the National Brain Tumor Foundation (NBTF) the death rate due to brain tumor has been increasing. This increase in deaths is caused due to many reasons like treatment not given on time, tumor not detected due to poor image quality or contrast and many more. The aim of this project is to avoid such situations and improve the quality of treatment. Machine learning has high dimensional features which can definitely be useful in such conditions. This will not only help in increasing accuracy but also help the doctors to cure the patient within the required time. The objective is to build a self-learning system which scans the images and detects the tumor.

Neural networks are nothing but a multilayer architecture which is used for quantitative analysis. Consider the neurons in our body they are a network of cells which perform functions as directed. The tasks performed in neural networks is similar to the task of neurons.

\author{
Dr. Dipti. Y. Sakhare \\ Guided by, Professor, Dept. of E\&TC, \\ MIT Academy of Engineering, Pune, India
}

\section{LITERATURE SURVEY}

Image processing is been used in the medical field for a very long time and for many tests. For detecting the brain tumor doctors suggest for tests like the positron emission tomography (PET), compute tomography (CT) and the most famous and excessively used is the magnetic resonance image (MRI). MRI is preferred by the majority because it does not emit harmful rays and has no side effects on the body moreover it gives a precise view of the disorder. When it comes to image processing there are many types of methods which are used to analyze or detect an image

While detecting any image many parameters are taken into consideration like the color, contrast, pixels, intensity, boundaries, dimension etc. On the basis of these parameters any image can be categorized and the optimal required function can be performed. For the detection of brain tumor many processes were adapted and with the development of technology many other processes and tests are being developed. Some of the processes which have been used are thresholding, region based, contour and shape, statistical based and machine learning but these methods came with their own demerits. In thresholding the pixels separated and then grouped together on the basis of their intensity values. In some cases, this method proved to be useful but, in some cases, where the tumor is at its very first stage the intensity or the color contrast of the lump formed is very fade and the pixels could be grouped

wrong showing no signs of tumor. The second method which was tested was the contour and shape method where boundaries and segments are detected in this the boundaries and segments of the lump were detected but as the lumps are of irregular sizes and sometimes they are formed near the inner lining of the brain due to which boundaries could merge and accuracy wasn't gained. In the third method which is the region-based method groups of similar regions and made and then further detected. Last method was statistical based which was based on probable values, labels and optimal distribution. After trials and tests of these methods machine learning came in the broad picture. Machine learning when further divided has a sub part called as deep learning which will be used in this project. The methods, the approach, its merits and demerits will be discussed further. 


\section{METHODOLOGY}

Deep Learning is a machine learning technique which involves networks with many layers to make predictions. These networks are in the headlines because of their exceptional results and accuracy. Before starting the implementation and seeing the magic of deep learning and neural networks it's very important to understand how these networks work and how to make them.

\section{1) Neural Network and its formation -}

The hypothesis of this theory started way before in the $18^{\text {th }}$ century by Alexander Bain (1873) and William James (1890). Through their work they both proposed that thoughts and activities in the body are nothing but interactions among the neuron and the brain. Similarly, this same theory can be used in machines and make a system which is self-learning. A neural network is a circuit of multiple neurons and nodes in artificial sense which is used to perform any desired task.

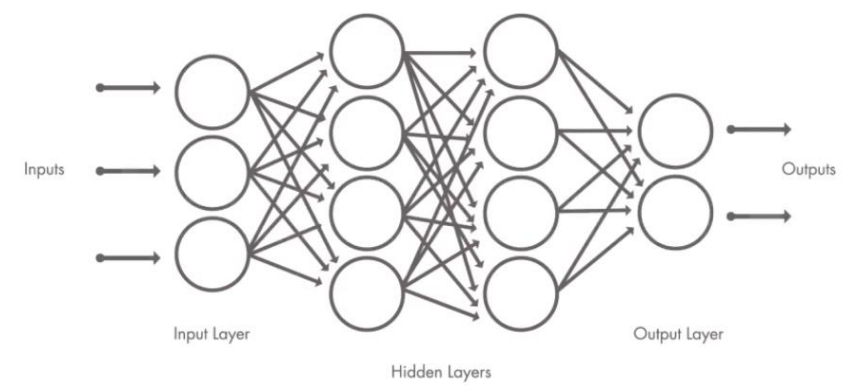

Fig.1.1. Basic Layout of a Neural Network

Neural networks are formed by multiple layers of information, tasks and functions but if one has to define a simple neural network it consists of mainly three layers which is the input layer, hidden layers and the output layers. As the name suggests the input layer is where the input command is given and the output layers is from which the required outcome is achieved. The hidden layer is where all the tasks and functions take place. Fig1.1 shows the structure of a very basic neural network. The network formed can be universal set which includes everything or it can be a sub set which is dedicated for a particular task. To detect any image, it is very important to train the network first and assign proper functions for the required task. In order to train a network for a set of images the pre-processing on the images necessary.

\section{2) Image Pre-processing -}

As mentioned before images can be classified into many sections and on the basis of many parameters. For training a network all the images which are being used or which are being stored should be uniform. Images can be distinguished on the basis of RGB and Gray-scale. The MRI scans are mostly in the gray scale format so all the images stored in the folder should preferably be in the gray scale format. The dimensions of the image should be uniform as well for which one should resize the image and change its dimensions to ' $227 \times 227$ ' which is the required dimension when a network is being formed. Fig.1.2. shows the image pre-processing for

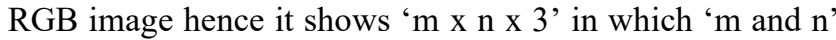
are the dimensions of the image.

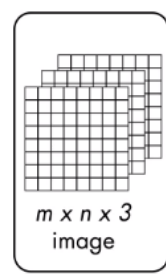

ge
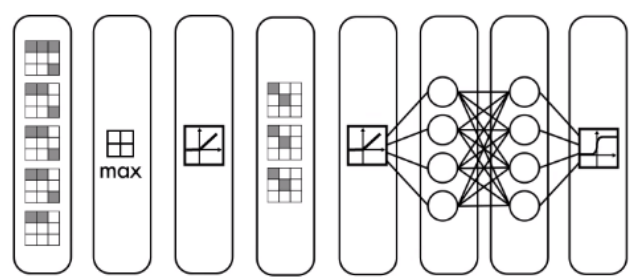

Fig.1.2. Image Pre-processing

\section{3) Transfer Network -}

While using deep learning we often come around pre-trained or trained networks which already have a defined data storage. In order to update the network or add new data a process is used which is called transfer network. In this process the changes are made in the input layer and the layer just before the outcome. Another important factor which plays a major role is the memory management. Transfer network also helps in maintaining the storage. As neural network is a self-learning system it stores only the required information in the dedicated folders and you don't land up with double images in the folder.

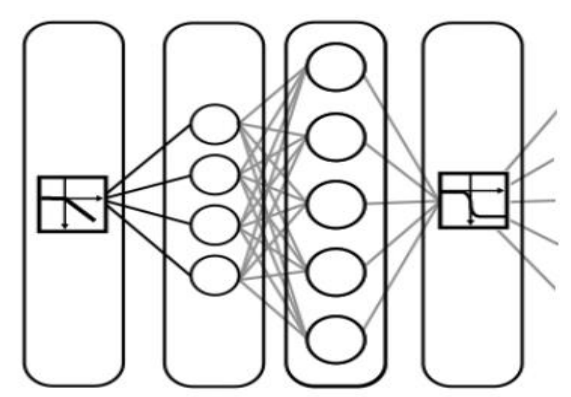

Fig.1.3. Transfer of Network

When you compare Fig.1.2 and Fig.1.3 you can notice the changes in networks. Fig.1.3 is nothing but the changes in the last layers of the same system which is showed in Fig.1.2. Once you have completed the process of transferring the network you have to again train the network with the new stored images.

\section{4) Network Training -}

The last task is to train the network with the given data set and the given commands. For detecting the brain tumors, the network had to be trained on numerous MRI scans and perform segmentation in order to detect the tumor accurately. Tumors are lump like structures which are formed in the brain. At times even multiple tumors are developed within the brain. The brain can be divided into three main parts which is the cerebrum, the brain stem and the cerebellum. Further the hemispheres of the brain are divided into four lobes which are the frontal lobe, parietal lobe, temporal lobe and the occipital lobe. These lobes work in conjunction to maintain the functions of the body and each lobe carries out multiple functions. The tumor can be formed 
in any of these lobes. The network will be trained for detecting the tumor and also detect in which part of the lobe it is formed. The input has to be a data set of multiple scans and the region of the lobes has to be defined.

\section{5) Results -}

The intension of this project was to obtain results similar to the results given by a specialist. There are many software available to perform deep learning but the most feasible would be MATLAB as there are inbuilt functions available like the image processing tool box and the deep learning tool box. One can practice or test functions on MATLAB's pretrained networks such as 'alexnet'. The circled part in Fig.1.4 and Fig.1.5 is the tumor which was detected after segmentation. The results show that with the help of this system single as well as multiple tumors can be detected with greater accuracy.

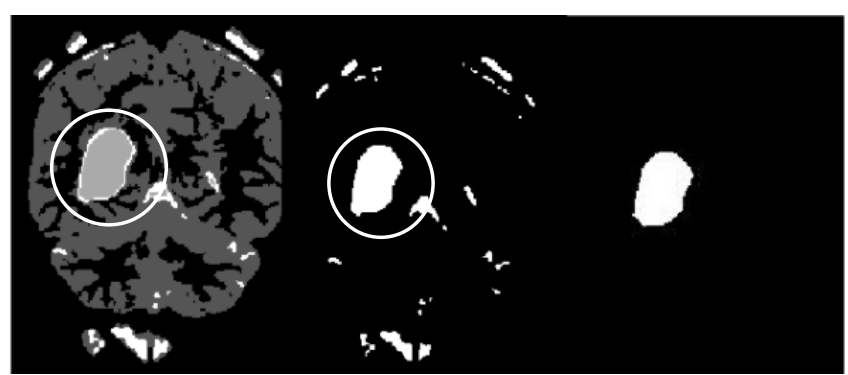

Fig.1.4. Detection of Brain Tumor
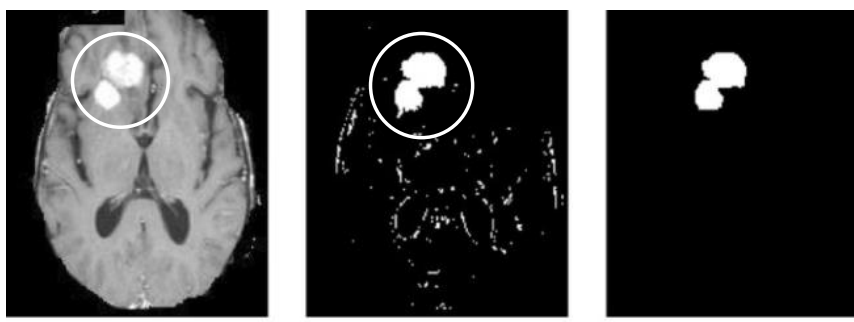

Fig.1.5. Detection of Brain Tumor

\section{CONCLUSION AND FUTURE SCOPE}

Image processing has found its way in the biomedical stream and will continue to grow. There is a wide perspective of using image processing for many other tests as well like detecting the hemoglobin, WBC and RBC in the blood. It can also be used for retinopathy which can detect diabetes and detect cataract as well. Adaptive systems like this have proved to be useful and can be further developed as well.

\section{REFERENCES}

[1] Tobias Christian Cahoon, Melanie A. Sutton, James C. Bezdek, Breast Cancer Detection Using Image Processing Techniques, Department of Compute Science University of Florida.

[2] Prabhjot Kaur Chahal, Shreelekha Pandey, Shivani Goel, A survey on brain tumor detection techniques for MR images, Springer.

[3] T Kalaiselvi, P Nagaraja, A rapid automatic brain tumor detection method for MRI images using modified minimum error thresholding technique, International Journal of Imaging Systems.

[4] Jin Liu, Min Li, Jianxin Wang, Fangxiang Wu, Tianming Liu, Y Pan, A survey of MRI based brain tumor segmentation methods, IEEE.

[5] M Prastawa, E Bullitt, S Ho, G Gerig, A brain tumor segmentation framework based on outlier detection, Medical Image Analysis.

[6] S Deepak, PM Ameer, Brain tumor classification using deep CNN features vis transfer learning, Volume 111 Science Direct. 